\title{
SPECTRAL MULTIPLICITY FOR TENSOR PRODUCTS OF NORMAL OPERATORS
}

\author{
EDWARD A. AZOFF ${ }^{1}$
}

\begin{abstract}
Two normal operators $N_{1}$ and $N_{2}$ are constructed such that for any pair $m_{1}$ and $m_{2}$ of their respective multiplicity functions, the 'convolution' $\left(m_{1} * m_{2}\right)(\lambda)$ $\equiv \Sigma\left\{m_{1}\left(\lambda_{1}\right) \cdot m_{2}\left(\lambda_{2}\right) \mid \lambda_{1} \cdot \lambda_{2}=\lambda\right\}$ fails to be a multiplicity function for the tensor product $N_{1} \otimes N_{2}$.
\end{abstract}

Introduction. All operators discussed in this paper will be normal operators acting on separable Hilbert spaces. Let $N$ be such an operator and let $\nu$ be one of its scalar spectral measures. The theory of spectral multiplicity associates with $N$ a function $m: \mathbf{C} \rightarrow \mathbf{N} \cup\{\infty\}$ such that the equivalence class [m], (i.e. the family of functions: $\mathbf{C} \rightarrow \mathbf{N} \cup\{\infty\}$ agreeing with $m$ off a $\nu$-null set) provides a complete unitary invariant for $N$. In particular, the spectrum of $N$ and the measure class of $\nu$ are completely determined by $[\mathrm{m}]_{\nu}$. On the other hand, it is only the equivalence class $[m]_{\nu}$ and not $m$, that is uniquely determined by $N$. It would be nice to choose 'canonical' representatives for these equivalence classes. For operators with countable spectra this is possible: we simply take $m$ to be the unique representative of its equivalence class which is supported on the point spectrum of $N$.

If $N_{i}(i=1,2)$ have countable spectra and the canonical multiplicity functions $m_{i}(i=1,2)$ are chosen as in the preceding paragraph, then the function $m_{1} * m_{2}$ defined by

$$
\left(m_{1} * m_{2}\right)(\lambda) \equiv \sum\left\{m_{1}\left(\lambda_{1}\right) \cdot m_{2}\left(\lambda_{2}\right) \mid \lambda_{1} \cdot \lambda_{2}=\lambda\right\}
$$

provides a multiplicity function for the tensor product $N_{1} \otimes N_{2}$. In their paper [1], M. B. Abrahamse and T. L. Kriete constructed 'canonical' multiplicity functions for multiplication operators. They then asked whether formula (1) continues to provide a multiplicity function for the tensor product of multiplication operators with 'canonical' multiplicity functions $m_{1}$ and $m_{2}$ respectively. Example 2 of this note settles this question negatively. One might think that this could be rectified by changing the Abrahamse-Kriete multiplicity function. Example 3 of this note exhibits two multiplication operators $N_{1}$ and $N_{2}$ such that for every choice of multiplicity functions, equation (1) fails to provide a multiplicity function for $N_{1} \otimes N_{2}$. This can be interpreted as meaning that there is, in general, no canonical way to choose representatives for multiplicity classes of normal operators.

Received by the editors November 12, 1979 and, in revised form, February 5, 1980.

AMS (MOS) subject classifications (1970). Primary 47B15.

${ }^{1}$ The author acknowledges partial support by the National Science Foundation. 
Notation and preliminaries. We recall the basic components of multiplicity theory for normal operators. For a fuller discussion, the reader should consult the introductory sections of [1] or [2].

Let $N$ be a normal operator with spectral measure $E$. A measure $\nu$ on $\mathbf{C}$ is said to be a scalar spectral measure for $N$ in case $E(S)=0$ if and only if $\nu(S)=0$ for $S$ a Borel set in C. (All measures discussed in this note are completions of probability measures defined on complete separable metric spaces.) There exists a direct integral $\int_{\mathbf{C}}^{\oplus} H(\lambda) d \nu$ of Hilbert spaces having $\nu$ as a scalar spectral measure for $N$ such that $N$ is unitarily equivalent to the operator $M$ on $\int_{\mathbf{C}}^{\oplus} H(\lambda) d \nu$ defined by $M f(\lambda)=\lambda f(\lambda)$. The function $m: \mathbf{C} \rightarrow \mathbf{N} \cup\{\infty\}$ defined by $m(\lambda)=\operatorname{dim} H(\lambda)$ is called a multiplicity function for $N$; its equivalence class $[m]_{v}$ is called the multiplicity class of $N$ and constitutes a complete unitary invariant for $N$.

Let $(X, \mu)$ be a measure space. As usual, we write $L^{\infty}(\mu)\left(L^{2}(\mu)\right)$ for the collection of equivalence classes of bounded measurable (respectively square-integrable) complex-valued functions on $X$. Let $\phi \in L^{\infty}(\mu)$. Then the multiplication operator $M_{\phi}$ is defined by $M_{\phi} f=\phi f\left(f \in L^{2}(\mu)\right)$. It is a bounded normal operator having $\nu \equiv \mu \circ \phi^{-1}$ as a scalar spectral measure. The papers [1] and [2] address the problem of constructing multiplicity functions for such operators. We close this section by summarizing these results.

If $\varphi$ is a Borel representative of $\phi$, we define $m_{\varphi}: \mathbf{C} \rightarrow \mathbf{N} \cup\{\infty\}$ by taking $m_{\varphi}(\lambda)$ to be the cardinality of the set $\varphi^{-1}(\lambda)$. (All infinite cardinalities are identified.)

Proposition 1. Let $\phi \in L^{\infty}(X, \mu)$, take $M_{\phi}$ to be the corresponding multiplication operator, and set $\nu=\mu \circ \phi^{-1}$.

(1) There is a Borel representative $\varphi_{0}$ of $\phi$ such that $m_{\varphi_{0}}$ is a multiplicity function for $M_{\phi}$.

(2) If $\varphi$ is any Borel representative of $\phi$, then $m_{\varphi}$ is $\nu$-measurable and $m_{\varphi}(\lambda)>$ $m_{\varphi_{0}}(\lambda)$ for $\nu$-almost all $\lambda \in \mathbf{C}$.

(3) Given a Borel multiplicity function $m$ for $M_{\phi}$, there is a Borel representative $\varphi$ of $\phi$ such that $m_{\varphi}(\lambda)<m(\lambda)$ for all $\lambda \neq 0$.

(4) In order that $M_{\phi}$ have uniform multiplicity one, it is necessary and sufficient that some (hence every) representative $\varphi$ of $\phi$ be one-to-one on a set of full $\mu$-measure.

Proof. Statements (1) and (2) constitute Theorem 4.1 of [2]; statement (4) is contained in Corollary 4.1 of that paper. To prove (3), choose $\varphi_{0}$ as in (1), and set $E=\left\{\lambda \in \mathbf{C} \mid m_{\varphi_{0}}(\lambda)>m(\lambda)\right\}$. Since $\nu(E)=0$, there is a Borel set $F$ containing $E$ with $\nu(F)=0$. Set

$$
\varphi(x)= \begin{cases}\varphi_{0}(x) & \text { if } x \notin \varphi_{0}^{-1}(F), \\ 0 & \text { if } x \in \varphi_{0}^{-1}(F) .\end{cases}
$$

For $\lambda \neq 0$, we have $\varphi^{-1}(\lambda) \subseteq \varphi_{0}^{-1}(\lambda)$ so $m_{\varphi}(\lambda)<m_{\varphi_{0}}(\lambda)$. Moreover, if $\lambda \in E \sim\{0\}$, $\varphi^{-1}(\lambda)=\varnothing$ so $m_{\varphi}(\lambda)=0$. This completes the proof.

REMARK. If $\nu$ is not totally atomic the Borel representative $\varphi$ of (3) can be chosen to satisfy $m_{\varphi} \equiv m$. 
Finally we recall the construction of the Abrahamse-Kriete multiplicity function [1] for $M_{\phi}$. Given $\lambda \in$ ess ran $\phi$, a point $x \in X$ is said to belong to the essential preimage, $\phi_{\text {ess }}^{-1}(\lambda)$, of $\phi$ at $\lambda$ in case

$$
\varliminf_{\delta \rightarrow 0} \frac{\mu\left(V \cap \phi^{-1}\left(B_{\delta}(\lambda)\right)\right)}{\mu\left(\phi^{-1}\left(B_{\delta}(\lambda)\right)\right)}
$$

is strictly positive for every neighborhood $V$ of $x$; here $B_{\delta}(\lambda)$ denotes the closed ball of radius $\delta$ about $\lambda$. The function $\bar{m}_{\phi}: \mathbf{C} \rightarrow \mathbf{N} \cup\{\infty\}$ (or simply $\bar{m}$ if $\phi$ is understood) is defined as

$$
\bar{m}(\lambda)= \begin{cases}\text { cardinality of } \phi_{\text {ess }}^{-1}(\lambda) & \text { if } \lambda \in \text { ess } \operatorname{ran} \phi, \\ 0 & \text { if } \lambda \notin \text { ess } \operatorname{ran} \phi .\end{cases}
$$

It is shown in [1] that $\bar{m}$ is a multiplicity function for $M_{\phi}$; in the sequel, it will be referred to as the Abrahamse-Kriete multiplicity function for $M_{\phi}$.

Noncanonicity of multiplicity functions. Given functions $m_{i}: \mathbf{C} \rightarrow \mathbf{N} \cup\{\infty\}(i=$ $1,2)$, we adopt equation (1) as the definition of $m_{1} * m_{2}$.

PROPOSITION 2. Let $N_{1}$ and $N_{2}$ be normal operators having multiplicity functions $m_{1}$ and $m_{2}$ respectively. Let $\nu$ be a scalar spectral measure for $N_{1} \otimes N_{2}$, and suppose $m$ is one of its multiplicity functions. Then $\left(m_{1} * m_{2}\right)(\lambda)>m(\lambda)$ for $\nu$-almost all $\lambda$.

Proof. Let $\nu_{i}$ be a scalar spectral measure for $N_{i}$; up to absolute continuity, the measure $\nu$ is given by $\nu(E)=\left(\nu_{1} \times \nu_{2}\right)\left\{\left(\lambda_{1}, \lambda_{2}\right) \in \mathbf{C} \times \mathbf{C} \mid \lambda_{1} \cdot \lambda_{2} \in E\right\}$. By lowering the values of $m_{i}$ on a $\nu_{i}$-null set, we can make $m_{1}$ and $m_{2}$ Borel measurable. Since this can only decrease the value of $\left(m_{1} * m_{2}\right)(\lambda)$, we may as well assume $m_{1}$ and $m_{2}$ are Borel to start with. Since every normal operator is unitarily equivalent to a multiplication operator, we may take $N_{i}$ to be a multiplication operator $M_{\phi_{1}}$ acting on a Hilbert space $L^{2}\left(X_{i}, \mu_{i}\right)$. Apply Proposition 1(3) to construct a Borel representative $\varphi_{i}$ of $\phi_{i}$ such that $m_{\varphi_{i}}(\lambda)<m_{i}(\lambda)$ for $\lambda \neq 0$, let $\varphi: X_{1} \times X_{2} \rightarrow \mathbf{C}$ by $\varphi\left(x_{1}, x_{2}\right)=\varphi_{1}\left(x_{1}\right) \cdot \varphi_{2}\left(x_{2}\right)$ and write $\phi=[\varphi]_{\mu_{1} \times \mu_{2}}$. Then $N_{1} \otimes N_{2}$ is unitarily equivalent to $M_{\phi}$ acting on $L^{2}\left(X_{1} \times X_{2}, \mu_{1} \times \mu_{2}\right)$ and so $m(\lambda)<m_{\varphi}(\lambda)$ for $\nu$-almost all $\lambda$ by Proposition 1(2). But for $\lambda \neq 0$, we have $m_{\varphi}(\lambda)=\left(m_{\varphi_{1}} * m_{\varphi_{2}}\right)(\lambda)<\left(m_{1} * m_{2}\right)(\lambda)$ so the proof is complete as long as $\{0\}$ is not an atom for $\nu$. On the other hand, if $\{0\}$ is an atom for $\nu$, then $m(0)=\operatorname{dim} \operatorname{ker}\left(N_{1} \otimes N_{2}\right)$ which is easily seen to be less than or equal to $m_{1} * m_{2}(0)$.

Proposition 2 means that $m_{1} * m_{2}$ can only fail to be a multiplicity function for $N_{1} \times N_{2}$ by being too large, i.e. by having the strict inequality $\left(m_{1} * m_{2}\right)(\lambda)>m(\lambda)$ hold on a set of $\lambda$ which does not have $\nu$ measure zero. The three examples which follow illustrate successively more pathological instances of this behavior.

In each of these examples, $\mu_{i}(i=1,2)$ is a measure supported on $[0,1], \varphi_{1}=\varphi_{2}$ is the identity function on $[0,1], \phi_{i}=\left[\varphi_{i}\right]_{\mu_{i}}$, and $N_{i}$ is the multiplication operator $M_{\phi_{i}}$ acting on $L^{2}\left([0,1], \mu_{i}\right)$. Thus $N_{1} \otimes N_{2}$ is unitarily equivalent to the multiplication operator $M_{\phi_{1} \otimes \phi_{2}}$ acting on $L^{2}\left([0,1] \times[0,1], \mu_{1} \times \mu_{2}\right)$. It will be convenient to let $p: \mathbf{R}^{2} \rightarrow \mathbf{R}$ denote multiplication. Of course, $\mu_{i}$ is a scalar spectral measure for $N_{i}$ and $\nu \equiv\left(\mu_{1} \times \mu_{2}\right) \circ p^{-1}$ provides a scalar spectral measure for $N_{1} \otimes N_{2}$. 
EXAMPLE 1. Let $\mu_{1}=\mu_{2}$ be the point-mass measure based at 1 , and let $m_{1}=m_{2}$ : $\mathbf{C} \rightarrow \mathbf{N}$ be the constant function 1 . Then $m_{i}$ is a multiplicity function for $N_{i}$, but $m_{1} * m_{2} \equiv \infty$ is not a multiplicity function for $N_{1} \otimes N_{2}=I$. On the other hand, $\bar{m}_{1}=\bar{m}_{2}$ equals the characteristic function of the singelton set $\{1\}$, so $\bar{m}_{1} * \bar{m}_{2}$ is a multiplicity function for $N_{1} \otimes N_{2}$.

EXAMPLe 2. Let $A_{1}=\left\{e^{-q} \mid q>0, q \in \mathbf{Q}\right\}$ and $A_{2}=\left\{e^{-q \sqrt{2}} \mid q>0, q \in \mathbf{Q}\right\}$. Let $\mu_{i}$ be a totally atomic measure on $[0,1]$ having $A_{i}$ as its set of atoms. Since $A_{i}$ is dense in $[0,1]$, the Abrahamse-Kriete multiplicity function $\bar{m}_{i}$ turns out to be the characteristic function $X_{[0,1]}$. Thus $\left(\bar{m}_{1} * \bar{m}_{2}\right)(\lambda)=\infty$ for each $\lambda \in(0,1)$. On the other hand, $\mu_{1} \times \mu_{2}$ is supported on $A_{1} \times A_{2}$ and $p$ is one-to-one on $A_{1} \times A_{2}$, so $N_{1} \otimes N_{2}$ has uniform multiplicity one. Thus $\bar{m}_{1} * \bar{m}_{2}$ is not a multiplicity function for $N_{1} \otimes N_{2}$, and we have a counterexample to the conjecture in $\$ 7$ of [1]. Note however that $m_{i} \equiv X_{A_{i}}$ provides a multiplicity for $N_{i}$ such that $m_{1} * m_{2}$ is a multiplicity function for $N_{1} \otimes N_{2}$.

EXAMPLE 3. We construct measures $\mu_{i}(i=1,2)$ such that for any choice $m_{i}$ of multiplicity function for $N_{i}, m_{1} * m_{2}$ fails to be a multiplicity function for $N_{1} \otimes N_{2}$. We need the following lemma.

LEMMA. There exist four uncountable, closed subsets $A, B, C, D$ of $[0,1]$ with the following properties:

(1) $p$ is one-to-one on $(A \times D) \cup(B \times C) \cup(B \times D)$.

(2) For each $b \in B$, there is a unique $d \in D$ such that $b \cdot d \in A \cdot C$ and for each $d \in D$, there is a unique $b \in B$, such that $b \cdot d \in A \cdot C$.

(3) $A \cdot C \subseteq B \cdot D$.

Proof. For a member $x \in[0,10]$, we let $x_{n}(n=0,1,2, \ldots)$ denote the $n$th digit in its decimal expansion. (Expansions ending in nines will not be considered.) Let $A_{0}, B_{0}, C_{0}, D_{0}$ be the sets of numbers having the decimal expansions indicated in the following table.

$\begin{array}{cccc} & x_{0} & x_{2 n-1}(n>0) & x_{2 n}(n>0) \\ A_{0} & 1 & 0 \text { or } 1 & 1 \\ B_{0} & 0 & 0 & 0,1,3 \text { or } 4 \\ C_{0} & 3 & 0 \text { or } 3 & 3 \\ D_{0} & 4 & 0,1,3 \text { or } 4 & 4-x_{2 n-1}\end{array}$

It is easy to check that $A_{0}, B_{0}, C_{0}, D_{0}$ satisfy conditions analogous to (1), (2) and (3) of the lemma with addition in place of multiplication. Set $A=\left\{e^{-x} \mid x \in A_{0}\right\}$, $B=e^{-B_{0}}, C=e^{-C_{0}}, D=e^{-D_{0}}$.

By Theorem 8.1 of [3], we know that the sets $A$ and $C$ can be equipped with nonatomic measures $\alpha$ and $\gamma$ respectively. Define a measure $\beta$ on $B$ by setting $\beta(S)=(\alpha \times \gamma)\left(p^{-1}(S \cdot D)\right)$. Similarly let $\delta$ be defined on $D$ by $\delta(S)=$ $(\alpha \times \gamma)\left(p^{-1}(B \cdot S)\right)$. Finally we take $\mu_{1}=(\alpha+\beta) / 2, \mu_{2}=(\gamma+\delta) / 2$.

We first show that $N_{1} \otimes N_{2}$ has uniform multiplicity 1 . To see this, let $E=B \times$ $D \cap p^{-1}(A \cdot C)$. By (2) of the lemma, each section $E_{d} \equiv\{b \in B \mid(b, d) \in E\}$ of $E$ in a singleton, and hence has $\mu_{1}$ measure zero. It follows by Fubini's theorem 
that $\mu_{1} \times \mu_{2}(E)=0$. By (1) and (3) of the lemma, $p$ is one-to-one on $(A \cup B) \times$ $(C \cup D) \sim E$. Since $\mu_{1} \times \mu_{2}$ is supported on this set, we conclude by Proposition 1(4) that $N_{1} \otimes N_{2}$ has uniform multiplicity one.

Suppose next that $m_{1}$ and $m_{2}$ are multiplicity functions for $N_{1}$ and $N_{2}$ respectively. Since $\mu_{i}$ is a scalar spectral measure for $N_{i}$, there is a set $X_{i}$ of full measure in $[0,1]$ on which $m_{i}$ is identically one. If $\lambda$ belongs to both of the sets $\left(X_{1} \cap A\right)$ $\cdot\left(X_{2} \cap C\right)$ and $\left(X_{1} \cap B\right) \cdot\left(X_{2} \cap D\right) \cap A \cdot C$, then $\left(m_{1} * m_{2}\right)(\lambda)>2$. We will show that these sets have full $\nu$-measure in $A \cdot C$. Now

$$
\nu(A \cdot C)=\left(\mu_{1} \times \mu_{2}\right)\left(p^{-1}(A \cdot C)\right)=\left(\mu_{1} \times \mu_{2}\right)(A \times C \cup E)=\frac{1}{4} .
$$

Thus we will know that $\left(m_{1} * m_{2}\right)(\lambda)>1$ on a set of positive $\nu$-measure, so that in view of the preceding paragraph, $m_{1} * m_{2}$ cannot be a multiplicity function for $N_{1} \otimes N_{2}$.

It remains to show that

$$
\nu\left[\left(X_{1} \cap A\right) \cdot\left(X_{2} \cap C\right)\right]=\nu\left[\left(X_{1} \cap B\right) \cdot\left(X_{2} \cap D\right) \cap A \cdot C\right]=\frac{1}{4} .
$$

The first set is easy: $\nu\left(\left(X_{1} \cap A\right) \cdot\left(X_{2} \cap C\right)\right)>\left(\mu_{1} \times \mu_{2}\right)\left[\left(X_{1} \cap A\right) \times\left(X_{2} \cap C\right)\right]$ $=\mu_{1}(A) \cdot \mu_{2}(C)=\frac{1}{4}$. To handle the second set, we first note that if $S \subseteq A \times C$, then $\left(\mu_{1} \times \mu_{2}\right)(S)=\frac{1}{4}(\alpha \times \gamma)(S)$. It follows that $\nu\left[\left(B \sim X_{1}\right) \cdot D \cap A \cdot C\right]=$ $\frac{1}{4}(\alpha \times \gamma)\left(p^{-1}\left[\left(B \sim X_{1}\right) \cdot D\right]\right)$ which is by definition of $\beta$ equal to $\frac{1}{4} \beta\left(B \sim X_{1}\right)$. Since $X_{1}$ has full measure, we conclude that $\nu\left[\left(B \sim X_{1}\right) \cdot D \cap A \cdot C\right]=0$. A similar argument shows that $\nu\left[B \cdot\left(D \sim X_{2}\right) \cap A \cdot C\right]=0$. Thus

$$
\nu\left[\left(X_{1} \cap B\right) \cdot\left(X_{2} \cap D\right) \cap A \cdot C\right]=\nu(B \cdot D \cap A \cdot C)=\nu(A \cdot C)=\frac{1}{4}
$$

as desired.

\section{REFERENCES}

1. M. B. Abrahamse and T. L. Kriete, The spectral multiplicity of a multiplication operator, Indiana Univ. Math. J. 22 (1973), 845-857.

2. E. Azoff and K. Clancey, Spectral multiplicity for direct integrals of normal operators, J. Operator Theory 3 (1980), 213-235.

3. K. R. Parthasarathy, Probability measures on metric spaces, Academic Press, New York, 1967.

Department of Mathematics, University of Georgia, Athens, Georgin 30602 